\title{
Application of Dosage Combinations of Evagrow Biofertilizer and Chemical Fertilizer on Soil Characteristics, Growth and Yield of Rice
}

\author{
I Nyoman Merit", I Wayan Narka, and Tatiek Kusmawati \\ Program Study of Agroecotechnology, Faculty of Agriculture, Udayana University \\ Bukit Jimbaran, Badung, Bali 80362 \\ *Corresponding author: nym_merit@yahoo.com
}

\begin{abstract}
Research on the effect of dosage combination of Evagrow bio-fertilizer and chemical fertilizers on soil properties, growth and yield of rice has been carried out in the glasshouse, Faculty of Agriculture, Udayana University using factorial experiment with a Randomized Block Design (RBD). The first factor is Evagrow bio-fertilizer in 3 level, i.e. E0 (without bio-fertilizers), E1 (5 g bio-fertilizer/L) E2 (10 grams bio-fertilizer/L). The second factor is chemical fertilizer, which consists of 3 levels: K0 (without chemical fertilizers), K1 (150 kg Urea/ha $+75 \mathrm{~kg} \mathrm{SP} 36 / \mathrm{ha}+37.5 \mathrm{~kg} \mathrm{KCl} / \mathrm{ha}$ ), K2 $(300 \mathrm{~kg} \mathrm{Urea} / \mathrm{ha}+150 \mathrm{~kg} \mathrm{SP} 36 / \mathrm{ha}+75 \mathrm{~kg} \mathrm{KCl} / \mathrm{ha})$. The results showed that chemical fertilizers give a significant and very significant effect on most of the rice growth and yield parameters. The application of chemical fertilizers K1 and K2 increased yield of dry grain harvest to $52.87 \%$ and $102.54 \%$ compared to controls. Application of Evagrow bio-fertilizer did not significantly increase growth and yield of rice. There is no interaction between chemical fertilizers and Evagrow biofertilizer. Similarly to some of the soil characteristics, biological fertilizer and chemical fertilizer application did not show significant effect on most of the soil characteristics, except on salt levels.
\end{abstract}

Keywords: Evagrow Biofertilizer, Inorganic Fertilizer, Oryza Sativa

\section{INTRODUCTION}

Rice (Oryza sativa L.) is the main source of carbohydrate around the world including Indonesia [1]. Demand on rice increase continuously of about 2.23 $\%$ per year [2]. Demand of rice increase continuously as population increased, however, this is not followed by increased rice production. Demand for rice reached 32 million ton while current national rice production was only 31.5 ton/ha [3]. Effort to increase rice production via technology development must be done to support food security in Indonesia.

Research on application of organic fertilizer such as cow manure, compost, worm manure and green manure has been done. Use of organic fertilizer alone, cannot increase productivity and maintain food security. Therefore, holistic nutrition approach which combine application of organic fertilizer and an-organic fertilizer to increase productivity and environmen-tal sustainability need to be done [4]. Research on the effect of organic fertilizer and an-organic fertilizer on rice growth and yield has been published at Agrivigor Journal, Hasanudin University, Makassar [5]. The use of bio-fertilizer is still limited. Research on the use of Nitrobine bio-fertilizer combine with compost and chemical fertilizer has been done by El-Nagar (2010) on flower plants for 2 seasons [6]. Results of their research shows that optimal dose was 15 ton compost/ha, 3g NPK inorganic fertilizer/pot/month on treatment employ-ing Nitrobine bio-fertilizer $10 \mathrm{~g} / \mathrm{pot}$ shows the best respond. It revealed that Nitrobine bio- fertilizer containing Azoto-bacter, Azospirillum and phosphate solving bacteria plays an important role in providing nutrition.

\section{RESEARCH METHOD}

Research was conducted at a green house, Faculty of Agriculture, Udayana University. Each pot contain $10 \mathrm{~kg}$ soil, keep watered for a week to make it muddy and then rice seedlings were planted. Research was conducted in factorial design using Randomized Completely Block Design. There are two factors were examined: Evagrow bio-fertilizer and chemical fertilizer. Evagrow biofertilizer treatment consists of 3 level: without Evagrow bio-fertilizer (EO), 5 g Evagrow bio-fertilizer (E1) and $10 \mathrm{~g}$ Evagrow bio-fertilizer per liter (E2). Chemical fertilizer consists of 3 level, i.e: without chemical fertilizer (K0), $150 \mathrm{~kg}$ Urea/ha $+75 \mathrm{~kg} \mathrm{SP36} / \mathrm{ha}$ + 37,5 kg KCl/ha (K1), $300 \mathrm{~kg} \mathrm{Urea/ha} \mathrm{+150} \mathrm{kg} \mathrm{SP36/ha}$ $+75 \mathrm{~kg} \mathrm{KCl} / \mathrm{ha}(\mathrm{K} 2)$. In total there are nine treatment combinations. Each combination consists of 3 replicates so that there are 27 trial pots.

Parameter observed include soil and plants aspect. Soil parameters were nitrogen level $(\mathrm{N})$, phosphorous $(\mathrm{P})$ and Potassium $(\mathrm{K})$, soil $\mathrm{pH}, \mathrm{C}$-organic and soil salt level. Growth parameters include: number of shoots, plant height, rice yield parameter include: productive shoots, dry grain weight at harvest, oven dried grain weight, oven dried of dry shoot weight, and oven dried root weight. Nitrogen level $(\mathrm{N})$, phosphorous $(\mathrm{P})$ and Potassium $(\mathrm{K})$, were analyzed using Bray 1 method, C-organic using 
Walkey \& Black method and soil salt level using electric conductometer. Materials needed in this experiment were rice paddy soil sample, rice seedlings, chemicals for soil analyses, Evagrow bio-fertilizer, and chemical fertilizer such as Urea $(45 \% \mathrm{~N}), \mathrm{SP} 36\left(36 \% \mathrm{P}_{2} \mathrm{O}_{5}\right), \mathrm{KCl}(60 \%$ $\mathrm{K}_{2} \mathrm{O}$ ). Equipment required were bucket for growing rice, soil screener, sprayer, oven, digital balance, soil analyses equipment.

\section{RESULTS AND ANALYSIS}

Based on variables observed on rice growth, it can be seen that chemical fertilizer gave significant and highly significat effect on plat height, number and dry weight of shoots. Observation on number of shoot at 56 DAP (days after planting) shows that highest number of shoots were on treatment K2 (23.89 shoots), followed by K1 (18.56 shoots) and lowest K0 (13.44 shoots) or increased $38.02 \%$ and $77.69 \%$ to control, respectively (Table 1). Increased of number of shoots, shoot dry weight and shoots height was triggered by applica-tion of Urea (46\% $\mathrm{N})$, SP36 $\left(\begin{array}{lllll}36 \% & \left.\mathrm{P}_{2} \mathrm{O}_{5}\right) & \mathrm{KCl} & (60 \% & \mathrm{K}_{2} \mathrm{O}\end{array}\right)$, which increased $\mathrm{N}, \mathrm{P}$ and $\mathrm{K}$ availability. Soil use in this experiment has low fertility; it N-total level was $0.120 \%$ (low), available-P was 1.77 ppm (very low), available-K was 57.28 ppm (very low). Application of Urea, SP36 and $\mathrm{KCl}$ fertiliser has increased $\mathrm{N}, \mathrm{P}$ and $\mathrm{K}$ availability so that increased rice paddy growth. Rice paddy shoots is an important indicator for rice paddy growth. Dry shoot weight was increased on treatment K1 (47.38\%) and K2
(91.27\%). Improvement on growth, particularly on number of shoots will increase number of productive shoots (Table 2). Average productive shoots number after application of chemical fertilizer was found highest of treatment K2 $(25.67 \%)$, which was significantly different with K1 (20.44\%) and K0 (14.78\%). Increased on number of productive shoots affected fruit weight per shoots. Dry seed weight increased $52.87 \%$ and $102.54 \%$ on treatment $\mathrm{K} 1$ and $\mathrm{K} 2$. This increased was caused by improved plant growth, particularly on seedling number.

Estimation of grain yield per hectare, with planting space $30 \mathrm{~cm}$ x $30 \mathrm{~cm}$ was $5.92 \mathrm{ton} / \mathrm{ha}$ on $\mathrm{K} 1$ and 7.84 ton/ha on K2, while for control was 3.97 ton/ha. This increase was due to fertilizer application which give impact to soil nutrition availability. Increased in nutrition availability improved rice paddy growth, more seedling growth and more productive shoots (Table 2), and resulting in increase on harvested dry grain yield per hectare (Fig. 1).

Statistical analyses show that chemical fertilizer and Evagrow bio-fertilizer and its interaction do not give significant impact on shoot/root. Average shoot/root on Evagrow bio-fertilizer treatment was found on E1 (6.69), decreased on E2 (6.00) and lowest on E (5.75), while average shoot/root on chemical application was highest on K1 (6.42) which was not significanty different with K0 (6.01 and K2 (6.00) (Table 2).

TABLE 1.

EFFECT OF EVAGROW BIOFERTILISER AND CHEMICAL FERTILIZER ON RICE PADDY GROWTH.

\begin{tabular}{|c|c|c|c|c|}
\hline $\begin{array}{l}\text { Treatment } \\
\text { /parameter }\end{array}$ & $\begin{array}{c}\text { Maximum vegetative plant } \\
\text { height } \\
(\mathrm{cm})\end{array}$ & $\begin{array}{c}\text { Number of vegetative } \\
\text { shoots } \\
\text { (maximum) }\end{array}$ & $\begin{array}{l}\text { Oven dried shoot } \\
\text { weight }(\mathrm{g})\end{array}$ & $\begin{array}{l}\text { Oven dried root } \\
\text { weight }(\mathrm{g})\end{array}$ \\
\hline E0 & $87.11 \mathrm{a}$ & $18.56 \mathrm{a}$ & $39.37 \mathrm{a}$ & $14.55 \mathrm{a}$ \\
\hline E1 & $85.56 \mathrm{a}$ & $18.22 \mathrm{a}$ & $40.03 \mathrm{a}$ & $12.34 \mathrm{a}$ \\
\hline E2 & $86.89 \mathrm{a}$ & $19.11 \mathrm{a}$ & $40.38 \mathrm{a}$ & $14.29 \mathrm{a}$ \\
\hline $5 \%$ LSD & - & - & - & - \\
\hline $\mathrm{K} 0$ & $82.1 \mathrm{a}$ & $13.44 \mathrm{a}$ & $27.31 \mathrm{a}$ & $9.18 \mathrm{a}$ \\
\hline $\mathrm{K} 1$ & $87.8 \mathrm{~b}$ & $18.56 \mathrm{~b}$ & $40.24 b$ & $13.15 \mathrm{a}$ \\
\hline $\mathrm{K} 2$ & $89.7 \mathrm{~b}$ & $23.89 \mathrm{c}$ & $52.23 \mathrm{c}$ & $18.84 \mathrm{~b}$ \\
\hline $5 \%$ LSD & 3.30 & 2,96 & 4,98 & 4,35 \\
\hline E0K0 & $82.3 \mathrm{ab}$ & $13.67 \mathrm{a}$ & $27.76 \mathrm{a}$ & $9.07 \mathrm{a}$ \\
\hline E0K1 & $89.0 \mathrm{~d}$ & $18.00 \mathrm{bc}$ & $40.54 \mathrm{~b}$ & $14.26 \mathrm{abc}$ \\
\hline E0K2 & $90.0 \mathrm{~d}$ & $24.00 \mathrm{~d}$ & $49.82 \mathrm{c}$ & $20.31 \mathrm{c}$ \\
\hline E1K0 & $80.0 \mathrm{ab}$ & $11.67 \mathrm{a}$ & $25.09 \mathrm{a}$ & $9.21 \mathrm{a}$ \\
\hline $\mathrm{E} 1 \mathrm{~K} 1$ & $87.7 \mathrm{~cd}$ & $19.67 \mathrm{c}$ & $40.85 \mathrm{~b}$ & $11.40 \mathrm{ab}$ \\
\hline E1K2 & $89.0 \mathrm{~d}$ & $23.33 \mathrm{~d}$ & $54.14 \mathrm{c}$ & $16.40 \mathrm{bc}$ \\
\hline E2K0 & $84.0 \mathrm{abc}$ & $15.00 \mathrm{ab}$ & $29.07 \mathrm{a}$ & $9.27 \mathrm{a}$ \\
\hline E2K1 & $86.7 \mathrm{bcd}$ & $18.00 \mathrm{bc}$ & $39.34 \mathrm{~b}$ & $13.79 \mathrm{abc}$ \\
\hline $\mathrm{E} 2 \mathrm{~K} 2$ & $90.0 \mathrm{~d}$ & $24.33 \mathrm{~d}$ & $52.73 \mathrm{c}$ & $19.82 \mathrm{c}$ \\
\hline Duncan & MDRS & MDRS & MDRS & MDRS \\
\hline
\end{tabular}

Note: Numbers that followed by same letter in the same column means it not significantly different on 5\% LSD and Duncan $5 \%$. 


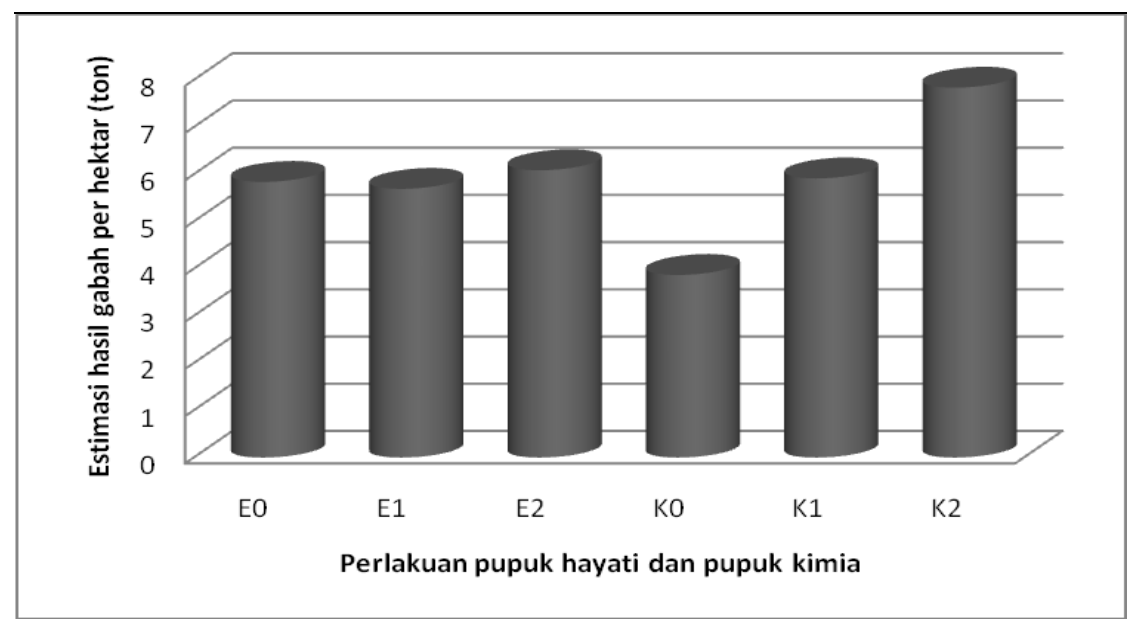

Fig. 1. Estimation of seed yield per hectare on after fertilizer treatment.

Evagrow application did not show significant impact on growth, yield and soil characteristics. This maybe caused by microbe that contained in Evagrow fertilizer was not active and do not grow well. Hight temperature on the glass house may cause less optimal growth of the soil microbia. This was shown on the average of rice paddy plants at 7 DAP to 56 DAP did not show significant effect, between E0, E1 and E2, rice paddy height was almost the same.

TABLE 2.

EFFECT OF EVAGROW BIO-FERTILIER AND CHEMICAL FERTILIZER ON RICE YIELD PARAMETER.

\begin{tabular}{|c|c|c|c|c|c|}
\hline $\begin{array}{l}\text { Treatmet } \\
\text { /parameter }\end{array}$ & $\begin{array}{c}\text { Number of } \\
\text { productive shoots }\end{array}$ & $\begin{array}{l}\text { Grain weight at } \\
\text { harvest }(\mathrm{g})\end{array}$ & $\begin{array}{l}\text { Oven-dried grain } \\
\text { weight }(\mathrm{g})\end{array}$ & $\begin{array}{l}\text { Estimated dry grain weight } \\
\text { at harvest/ha (ton) }\end{array}$ & Shoot/root \\
\hline E0 & $20.33 \mathrm{a}$ & $52.57 \mathrm{a}$ & $40.11 \mathrm{a}$ & $5.84 \mathrm{a}$ & $5.75 \mathrm{a}$ \\
\hline E1 & $20.11 \mathrm{a}$ & $51.24 \mathrm{a}$ & $38.83 \mathrm{a}$ & $5.69 \mathrm{a}$ & $6.69 \mathrm{a}$ \\
\hline $\mathrm{E} 2$ & $20.44 \mathrm{a}$ & $54.79 \mathrm{a}$ & $42.09 \mathrm{a}$ & $6.09 \mathrm{a}$ & $6.00 \mathrm{a}$ \\
\hline $5 \%$ LSD & - & - & - & & - \\
\hline K0 & $14.78 \mathrm{a}$ & $34.82 \mathrm{a}$ & $26.43 \mathrm{a}$ & $3.87 \mathrm{a}$ & $6.01 \mathrm{a}$ \\
\hline K1 & $20.44 \mathrm{~b}$ & $53.24 \mathrm{~b}$ & $40.69 \mathrm{~b}$ & $5.92 \mathrm{~b}$ & $6.42 \mathrm{a}$ \\
\hline K2 & $25.67 \mathrm{c}$ & $70.53 \mathrm{c}$ & $53.92 \mathrm{c}$ & $7.84 \mathrm{c}$ & $6.00 \mathrm{a}$ \\
\hline $5 \%$ LSD & 2,40 & 5,95 & 4,49 & 0,66 & - \\
\hline E0K0 & $16.00 \mathrm{a}$ & $35.8 \mathrm{a}$ & $27.50 \mathrm{a}$ & $3.98 \mathrm{a}$ & $6.07 \mathrm{a}$ \\
\hline E0K1 & $19.67 \mathrm{~b}$ & $52.7 \mathrm{~b}$ & $40.11 \mathrm{~b}$ & $5.86 \mathrm{~b}$ & $5.93 \mathrm{a}$ \\
\hline E0K2 & $25.33 \mathrm{c}$ & $69.2 \mathrm{c}$ & $52.72 \mathrm{c}$ & $7.68 \mathrm{c}$ & $5.26 \mathrm{a}$ \\
\hline E1K0 & $13.33 \mathrm{a}$ & $32.3 \mathrm{a}$ & $24.24 \mathrm{a}$ & $3.59 \mathrm{a}$ & $5.75 \mathrm{a}$ \\
\hline E1K1 & $21.67 \mathrm{~b}$ & $53.6 \mathrm{~b}$ & $40.92 \mathrm{~b}$ & $5.96 \mathrm{~b}$ & $7.33 \mathrm{a}$ \\
\hline E1K2 & $25.33 \mathrm{c}$ & $67.8 \mathrm{c}$ & $51.33 \mathrm{c}$ & $7.53 \mathrm{c}$ & $6.99 \mathrm{a}$ \\
\hline $\mathrm{E} 2 \mathrm{~K} 0$ & $15.00 \mathrm{a}$ & $36.3 \mathrm{a}$ & $27.53 \mathrm{a}$ & $4.04 \mathrm{a}$ & $6.22 \mathrm{a}$ \\
\hline E2K1 & $20.00 \mathrm{~b}$ & $53.4 \mathrm{~b}$ & $41.03 \mathrm{~b}$ & $5.93 \mathrm{~b}$ & $6.01 \mathrm{a}$ \\
\hline E2K2 & $26.33 \mathrm{c}$ & $74.7 \mathrm{c}$ & $57.69 \mathrm{c}$ & $8.29 \mathrm{c}$ & $5.76 \mathrm{a}$ \\
\hline Duncan & MDRS & MDRS & MDRS & MDRS & - \\
\hline
\end{tabular}

Note: Numbers that followed by same letter in the same column means it not significantly different on 5\% LSD and Duncan $5 \%$. 
TABLE 3.

EFFECT OF EVAGROW BIO-FERTILIZER AND CHEMICAL FERTILIZER ON A NUMBER OF SOIL PROPERTIES.

\begin{tabular}{|c|c|c|c|c|c|c|}
\hline $\begin{array}{l}\text { Treatment } \\
\text { /parameter }\end{array}$ & $\begin{array}{c}\text { N-total } \\
(\%)\end{array}$ & $\begin{array}{c}\text { Available P } \\
(\mathrm{ppm})\end{array}$ & $\begin{array}{c}\text { Available K } \\
(\mathrm{ppm})\end{array}$ & $\begin{array}{c}\text { C-organic } \\
(\%)\end{array}$ & $\begin{array}{l}\text { Soil level (mmhos } \\
\text { /cm) }\end{array}$ & $\begin{array}{l}\text { Soil } \\
\mathrm{pH}\end{array}$ \\
\hline E0 & $0,23 \mathrm{a}$ & $3,86 \mathrm{a}$ & $88,18 \mathrm{a}$ & $3,51 \mathrm{a}$ & $0,72 \mathrm{a}$ & $6,94 \mathrm{a}$ \\
\hline E1 & $0,24 \mathrm{a}$ & $3,90 \mathrm{a}$ & $88,09 \mathrm{a}$ & $3,34 \mathrm{a}$ & $0,75 \mathrm{a}$ & $6,92 \mathrm{a}$ \\
\hline E2 & $0,24 \mathrm{a}$ & $4,09 \mathrm{a}$ & $89,82 \mathrm{a}$ & $3,27 \mathrm{a}$ & $0,73 \mathrm{a}$ & $6,97 \mathrm{a}$ \\
\hline $5 \%$ LSD & - & - & - & - & - & - \\
\hline $\mathrm{K} 0$ & $0,22 \mathrm{a}$ & $3,53 \mathrm{a}$ & $87,31 \mathrm{a}$ & $3,18 \mathrm{a}$ & $0,56 \mathrm{a}$ & $6,96 \mathrm{a}$ \\
\hline K1 & $0,23 \mathrm{a}$ & $4,11 \mathrm{a}$ & $89,30 \mathrm{a}$ & $3,37 \mathrm{a}$ & $0,71 \mathrm{a}$ & $6,95 \mathrm{a}$ \\
\hline $\mathrm{K} 2$ & $0,26 \mathrm{a}$ & $4,22 \mathrm{a}$ & $89,48 \mathrm{a}$ & $3,58 \mathrm{a}$ & $0,93 \mathrm{~b}$ & $6,93 \mathrm{a}$ \\
\hline $5 \%$ LSD & - & - & - & - & 0,21 & - \\
\hline E0K0 & $0,21 \mathrm{a}$ & $3,47 \mathrm{a}$ & $84,39 \mathrm{a}$ & $2,99 \mathrm{a}$ & $0,56 \mathrm{a}$ & $6,95 \mathrm{a}$ \\
\hline E0K1 & $0,23 \mathrm{a}$ & $4,04 \mathrm{a}$ & 89,99 a & 3,66 a & $0,69 \mathrm{ab}$ & $6,91 \mathrm{a}$ \\
\hline E0K2 & $0,25 \mathrm{a}$ & $4,08 \mathrm{a}$ & 90,16 a & $3,90 \mathrm{a}$ & $0,90 \mathrm{bc}$ & $6,96 \mathrm{a}$ \\
\hline $\mathrm{E} 1 \mathrm{~K} 0$ & $0,23 \mathrm{a}$ & $3,41 \mathrm{a}$ & $86,78 \mathrm{a}$ & $3,27 \mathrm{a}$ & $0,58 \mathrm{a}$ & $6,96 \mathrm{a}$ \\
\hline E1K1 & $0,23 \mathrm{a}$ & $4,09 \mathrm{a}$ & 88,19 a & $3,31 \mathrm{a}$ & $0,74 \mathrm{ab}$ & $6,92 \mathrm{a}$ \\
\hline $\mathrm{E} 1 \mathrm{~K} 2$ & $0,26 \mathrm{a}$ & $4,19 \mathrm{a}$ & 89,31 a & $3,43 \mathrm{a}$ & $0,95 \mathrm{c}$ & $6,88 \mathrm{a}$ \\
\hline E2K0 & $0,21 \mathrm{a}$ & $3,70 \mathrm{a}$ & 90,77 a & $3,27 \mathrm{a}$ & $0,56 \mathrm{a}$ & $6,95 \mathrm{a}$ \\
\hline E2K1 & $0,23 \mathrm{a}$ & $4,20 \mathrm{a}$ & $89,73 \mathrm{a}$ & $3,13 \mathrm{a}$ & $0,69 \mathrm{ab}$ & $7,01 \mathrm{a}$ \\
\hline E2K2 & $0,28 \mathrm{a}$ & $4,39 \mathrm{a}$ & $88,97 \mathrm{a}$ & $3,41 \mathrm{a}$ & $0,93 \mathrm{c}$ & $6,95 \mathrm{a}$ \\
\hline Duncan & - & - & - & - & MDRS & - \\
\hline
\end{tabular}

Note: Numbers followed by same letters in the same column shows non-significant at 5\% LSD or 5\% DMRT (Duncan Multiple Range Test).

The same thing occurred on other growth parameters such as number of shoot and oven dried shoot weight, did not show significant effect (Table 1). Effect of Evagrow bio-fertilier did not show significant effect on rice paddy growth parameter, also on number of reproduc-tive shoots. Average number of productive shoot. Evagrow bio-fertilizer was found highest on E2 (20.33), decreased but not significant on E0 (20.33 and lowest on E1 (20.11). This maybe due to soil microbia content on biofertilizer, did not develop on soil so that cannot increase soil nutrition availability

Analyses on a number of soil properties including $\mathrm{N}$ total, Available-P, available-K after chemical fertilizer application revealed an increasing tendency but did not statistically significant. This may due to soil samples was collected at the end of the research. Nutrition that has been given may has decreased its availability. This was because Urea, SP36 and KCL applied was in salt form. Average salt level on chemical fertilizer was found highest on $\mathrm{K} 2$ treatment $(0,93 \mathrm{mmhos} / \mathrm{cm})$, significantly different with $\mathrm{K} 1 \quad(0,71 \mathrm{mmhos} / \mathrm{cm})$ and $\mathrm{K} 0 \quad(0,56$ mmhos/cm (Table 3 ).

Combination between natural fertilizer and chemical fertilizer was expected to have significant interaction, because with the increased of nitrogen, phosphorous and potassium availability will give favorable condition for microbial growth. But in this experiment, interaction between Evagrow bio-fertilizer and chemical fertilizer did not happen. This is because microbial on Evagrow biofertilizer could not grow well, although nitrogen, phosphorus and potassium availability has increased.

\section{CONCLUSION}

1. Application of Evagrow biofertilizer did not show significant impact on soil characteris-tics, growth and yield.

2. Application of chemical fertilizer showed significant impact on almost all growth and yield parameter. Application of $150 \mathrm{~kg} \mathrm{Urea} / \mathrm{ha}+75 \mathrm{~kg} \mathrm{SP} 36 / \mathrm{ha}+37,5$ $\mathrm{kg} \mathrm{KCl} / \mathrm{ha}(\mathrm{K} 1)$ and $300 \mathrm{~kg}$ Urea/ha $+150 \mathrm{~kg}$ $\mathrm{SP} 36 / \mathrm{ha}+75 \mathrm{~kg} \mathrm{KCl} / \mathrm{ha}(\mathrm{K} 2)$ able to increase harvest yield, each $52.87 \%$ and $102.54 \%$ compared to control.

3. Applicaton of chemical fertiliser did not show significant impac on soil structure, except for salt level.

4. There is no interaction between Evagrow biofertilizer with chemical fertilizer on all parameter observed.

Recommendation

It is recommended to continue with field research or set up a trial on difference bio-fertilizer to find out the effect of biofertilizer on plant growth and soil structure.

\section{ACKNOWLEDGMENT}

The authors would like to thank LPPM Udayana University for the research grant.

\section{REFERENCES}

[1] Saragih, B. 2001. Keynote Address Ministers of Agriculture Government of Indonesia, 2nd National Workshop On Strengthening The Development And Use Of Hybrid Rice In Indonesia 1:10. 
[2] Arafah and M. P. Sirappa 2003. Kajian penggunaan jerami dan pupuk $\mathrm{N}$, P, dan K pada lahan sawah irigasi. BPTP Sulawesi Selatan. Jurnal Ilmu Tanah dan Lingkungan 4(1):15-24.

[3] Darma, M.D.I. 2007. Swasembada Beras Se-buah Impian? Available: http://www.bali post.co.id/balipostceta/2007/9/17/o2.htm.

[4] Suriadikarta, D.A. and R.D.M. Simanungkalit. 2006. Pupuk Organik dan Pupuk Hayati, Organik Fertilizer and Biofertili-zer. Bogor: Balai Besar Penelitian dan Pengembangan Sumberdaya Lahan Pertanian, p. 312.
[5] Arafah. 2005. Pengaruh Pemberian Pupuk Organik dan Anorganik terhadap Pertum-buhan dan Hasil Padi Sawah. Jurnal Agrivigor 4(2).

[6] El-Nagar, A.H. 2010. Effect Biofertilizer, organik compost and mineral fertilizers on the growth, flowering and bulbs produc-tion of Narcissus tazetta, Journal Agricul-ture and Environmental Science 9(1). 